\title{
CRITICAL BEHAVIOR OF AN IGNITION MODEL IN CHEMICAL COMBUSTION
}

\author{
BY
}

PETER J. TONELLATO

Marquette University, Milwaukee, Wisconsin

\begin{abstract}
A model for the hot slab ignition problem is analyzed to determine critical conditions. The system is said to be super-critical if the solution of the reduced perturbation problem blows up in small finite time or sub-critical if the blow up time is large. Comparison principles for integral equations are used to construct upper and lower solutions of the equation. All solutions depend on two parameters $\varepsilon^{-1}$, the Zeldovitch number and $\lambda$, the scaled hot slab size. Upper and lower bounds on a 'critical' curve $\lambda_{c}(\epsilon)$ in the $(\epsilon, \lambda)$ plane, separating the super-critical from the sub-critical region, are derived based upon the lower and upper solution behavior. Numerical results confirm the parameter space analysis.
\end{abstract}

1. Introduction. Knowledge of events leading to ignition is fundamental to the science of combustion. A thorough review of ignition phenomena was presented by Merzhanov and Averson [11]. A singular perturbation approach known as activation energy asymptotics has been used in the analysis of ignition event models [13]. In particular, boundary-applied external source ignition has been studied in great detail $[5,6,7,12]$. Liñan and Kindelan [8] used this technique to study an embedded, symmetric 'hot spot' ignition event.

The goal of this paper is to present an activation energy asymptotic analysis of a model of the thermal ignition of a solid or gaseous reactant caused by a hot, inert slab of material embedded within a cool reactive medium. Thermal ignition of a reactive medium is a threshold phenomenon resulting from the extremely sensitive dependence of the reaction rate on temperature. In this scenario, a local rise in the temperature due to the inert heat source may cause ignition if heat-dissipation mechanisms do not overcome the local temperature increase due to the reaction. The interplay between these mechanisms determines the dynamics of the reaction and is the focal point of this analysis.

The mathematical model of the temperature profile is derived from the system of conservation laws used to model combustion dynamics. The simplification process includes the assumptions of single reactant and oxidizer, one-step kinetics, and the constant density approximation (for a complete development see Williams [13]). The

Received July 6, 1990.

(C)1991 Brown University 
resulting mathematical model in one space dimension is

$$
\frac{\partial T}{\partial t}=\frac{\partial^{2} T}{\partial x^{2}}+A(x) \exp (-E / R T), \quad x \in \mathbf{R}, \quad x \neq \pm a, t>0,
$$

where, $T(x, t)$ is the temperature, $E$ is the activation energy, $R$ is the ideal gas law constant and $A(x)$ is a pre-exponential factor incorporating both heat release and chemical concentration. Here, $A(x)=0$ for $|x|<a$ and $A(x)=A$ for $|x|>a$. The nonlinear term will be denoted by $\Omega(T)$. The initial condition for the hot slab ignition problem is

$$
\begin{aligned}
T(x, 0) & =T_{o}(x) \\
& =T_{i}(H(x+a)-H(x-a))+T_{o}(H(-x-a)+H(x-a)),
\end{aligned}
$$

where $H$ is Heaviside's function, $T_{i}$ represents the temperature of the hot inert slab, $T_{o}$ the temperature of the surrounding premixed fuel and oxidizer $T_{i}>T_{o}$ and $2 a$ is the width of the hot slab. The appropriate boundary conditions are

$$
\begin{aligned}
T( \pm \infty, t) & =T_{o}, \\
T\left( \pm a^{-}, t\right) & =T\left( \pm a^{+}, t\right)
\end{aligned}
$$

and

$$
\frac{\partial T}{\partial x}\left( \pm a^{-}, t\right)=\frac{\partial T}{\partial x}\left( \pm a^{+}, t\right)
$$

where (1.3a) is imposed for consistency, (1.3b) represents the continuity of temperature across the two interfaces of the slab and (1.3c) represents the continuity of heat flux across the interfaces.

In Sec. 2, activation energy asymptotics is employed in the analysis of the hot slab ignition model. The analysis indicates that the critical behavior can be related to the solution of an integral equation. This solution represents a perturbation from the initial temperature at the surface of the hot slab. Liñan and Williams [7] used asymptotic analysis to derive an integral equation of this type. Numerical manipulation of the equation resulted in predictions of the ignition time. These predictions depend on activation energy and the difference between inert and surface initial temperatures. The integral equation derived here is similar to that of Liñan and Kindelan [8]. They studied a spherically or cylindrically symmetric hot spot. The local curvature is the relevant geometric parameter thus, a scaling that takes into account the local curvature is sufficient for the ignition analysis. Here, on the other hand, we consider a 1-D slab geometry; the width of the slab is the parameter governing ignition. Our model is scaled in such a way that the effect of heat loss through both interfaces is incorporated into the resultant perturbation system. This is another step towards the analysis of ignition based on size rather than local geometric properties of a hot spot.

The critical behavior thus related to the solution of a nonlinear singular Volterra integral equation, it is possible to employ maximum principles to qualitatively analyze the solution and determine the super- and sub-critical conditions. Comparison theorems for Volterra equations are presented in Sec. 3. These results are used to 
construct bounding upper and lower solutions. Since the solution depends on the parameters of the problem, the upper and lower bounding solutions must do so as well. It is shown that should the parameter values lie in one region of the space, the upper and lower solutions determine that the perturbation must stay bounded for a relatively long time (sub-critical condition), an indication that ignition does not take place. On the other hand, if the values fall in another region of parameter space, the upper and lower solution dictate that the true solution will blow up in small finite time (super-critical condition), implying that ignition does occur. We denote the 'critical' curve separating these two regions by $\lambda_{c}(\epsilon)$, where $\lambda$ and $\epsilon$ are the appropriately defined parameters of the problem.

In Sec. 4 we discuss the product integration numerical technique. This technique accounts for both nonlinearities and singularity of the Volterra equation. Analysis of the algorithm, applied to equations whose solutions blow up in finite time, does not appear in the literature. We show that the algorithm accurately predicts blow up in finite time in a simple case, lending confidence to its ability to track the unique singular solution. A comparison between analytical bounds and the calculated critical curve, $\lambda_{c}(\epsilon)$, is discussed.

2. Asymptotics. Models of combustion dynamics such as (1.1) are difficult to analyze due in part to the nonlinear dependence on temperature in the Arrhenius term, $\Omega(T)$. This obstacle is partially overcome by the careful application of the method of activation energy asymptotics (A.E.A.).

One of the steps taken in A.E.A. is the determination of a reference temperature $T_{a}$. In many steady state and quasi-steady state problems the reference temperature is the adiabatic flame temperature. In those cases, the temperature field is never larger than the adiabatic temperature. Therefore, the reaction rate $\Omega(T)$, need only be defined for temperatures less than this reference temperature. The reaction rate function which approximates $\Omega(T)$ when $\varepsilon=\left(R T_{a}^{2} / E\right)$ is small is

$$
\Omega(T) \simeq A \exp \left(-E / R T_{a}\right) \exp \left(-\frac{E}{R T_{a}^{2}}\left(T_{a}-T\right)\right)=A^{\prime} \exp \left[-\frac{1}{\epsilon}\left(T_{a}-T\right)\right],
$$

where $A^{\prime}=A \exp \left(-E / R T_{a}\right)$. This approximation is valid for all space $x$, and time $t$. Note that for temperatures $T$ less than $T_{a}$, yet such that $T_{a}-T$ is not small, the approximate reaction rate can be taken to be zero. However, when $T-T_{a}$ is $O(\epsilon)$, the reaction rate is $O(1)$.

In cases of evolutionary temperature profiles, such as the ignition problem, the reference temperature must be carefully chosen. The natural choice for the hot slab ignition problem is the temperature at the interface $x= \pm a$, when $t=0^{+}$. In this problem the approximation to the Arrhenius term will be valid for temperatures below the reference temperature. However, one should recognize that the temperature may rise well above the reference temperature. In fact, when ignition occurs this will happen fairly quickly at which time the approximation and the model cease to be alid. In this paper we will determine the conditions under which ignition, in this ense, occurs. 
Formulation of inner and outer problems. The model, with the approximate reaction rate, now lends itself directly to singular perturbation analysis. The first step in the development is to solve the inert problem ((1.1) with $A$ set equal to zero). This problem is easily solved (c.f. Carslaw and Jaeger [2]) and we denote its solution by $T_{I}(x, t)$,

$$
\begin{aligned}
T_{I}(x, t)=\frac{T_{i}}{2} & {\left[\operatorname{erf}\left(\frac{a-x}{2 \sqrt{t}}\right)+\operatorname{erf}\left(\frac{a+x}{2 \sqrt{t}}\right)\right] } \\
& +\frac{T_{o}}{2}\left[\operatorname{erfc}\left(\frac{a-x}{2 \sqrt{t}}\right)+\operatorname{erfc}\left(\frac{a+x}{2 \sqrt{t}}\right)\right],
\end{aligned}
$$

where $\operatorname{erf}(z)$ and $\operatorname{erfc}(z)$ represent the error and complementary error functions, respectively. We use this inert temperature profile as the zeroth order term in an asymptotic expansion of the nondimensional temperature defined as

$$
\begin{aligned}
U(x, t) & \equiv T(x, t) /\left(T_{i}-T_{a}\right) \\
& \equiv \begin{cases}U_{I}^{(0)}(x, t)+\epsilon U_{I}^{(1)}(x, t)+\epsilon^{2} U_{I}^{(2)}(x, t)+\ldots & \text { for }|x|<a, \\
U_{A}^{(0)}(x, t)+\epsilon U_{A}^{(1)}(x, t)+\epsilon^{2} U_{A}^{(2)}(x, t)+\ldots & \text { for }|x|>a,\end{cases}
\end{aligned}
$$

where the reference temperature mentioned earlier is chosen to be

$$
\left.T_{a} \equiv T_{I}\left(x, 0^{+}\right)\right|_{x=a}=\left(T_{i}+T_{o}\right) / 2 \text {, }
$$

and

The zeroth order terms are

$$
\varepsilon=\frac{R T_{a}^{2}}{E\left(T_{i}-T_{a}\right)}
$$

$$
U_{I}^{(0)}(x, t) \equiv T_{I}(x, t) /\left(T_{i}-T_{a}\right) \quad \text { for }|x|<a
$$

and

$$
U_{A}^{(0)}(x, t) \equiv T_{I}(x, t) /\left(T_{i}-T_{a}\right) \quad \text { for }|x|>a .
$$

The subscript $I$ denotes the inert region located on the interval $|x|<a$ while the subscript $A$ denotes the reactive region located on the interval $|x|>a$.

A meaningful reduced problem can best be derived if first the spatial and temporal variables $x$ and $t$ are scaled appropriately. A scaling factor $\eta$ is defined as $\eta \equiv$ $\Omega\left(T_{a}\right) /\left(T_{i}-T_{a}\right)$. Introducing the scale transformation $x=\eta^{-1 / 2} x^{\prime}$ and $t=\eta^{-1} t^{\prime}$ and using (2.3) we derive the scaled first-order problem

$$
\frac{\partial U_{I}^{(1)}}{\partial t}=\frac{\partial^{2} U_{I}^{(1)}}{\partial x^{2}}, \quad|x| \leq \lambda
$$

and

$$
\frac{\partial U_{A}^{(1)}}{\partial t}=\frac{\partial^{2} U_{A}^{(1)}}{\partial x^{2}}+\frac{1}{\epsilon} \exp \left[U_{A}^{(1)}(x, t)-\frac{1}{\epsilon} \theta(x, t ; \lambda)\right], \quad|x|>\lambda,
$$

where $\lambda=\eta^{1 / 2} a, \theta(x, t ; \lambda)=\operatorname{erf}\left(\frac{x-\lambda}{2 \sqrt{t}}\right)+\operatorname{erfc}\left(\frac{x+\lambda}{2 \sqrt{t}}\right)$ and the primes have been dropped for convenience. In summary, we note that $U_{I}^{(1)}$ represents the perturbation from the inert temperature profile within the hot spot, while $U_{A}^{(1)}$ represen 1 this perturbation in the reactive region. 
The perturbation model can now be analyzed by a classical matched asymptotic analysis. Note that the problem is symmetric about $x=0$. This fact can be utilized by restricting the study of the system to the region $x \geq 0$ and imposing the boundary condition

$$
\left.\left[\frac{\partial U_{I}^{(1)}(x, t)}{\partial x}\right]\right|_{x=0}=0, \quad t>0 .
$$

The appropriate auxiliary conditions are

$$
\begin{gathered}
U_{I}^{(1)}(x, 0)=0, \quad 0<x<\lambda, \\
U_{A}^{(1)}(x, 0)=0, \quad \lambda<x<\infty, \\
{\left.\left[\frac{\partial U_{I}^{(1)}(x, t)}{\partial x}\right]\right|_{x=0}=0, \quad t>0,} \\
U_{A}^{(1)}(+\infty, 0)=0, \quad t>0,
\end{gathered}
$$

with transition conditions,

$$
U_{I}^{(1)}\left(\lambda^{-}, t\right)=U_{A}^{(1)}\left(\lambda^{+}, t\right), \quad t>0,
$$

and

$$
\left.\frac{\partial U_{I}^{(1)}(x, t)}{\partial x}\right|_{x=\lambda^{-}}=\left.\frac{\partial U_{A}^{(1)}(x, t)}{\partial x}\right|_{x=\lambda^{+}}, \quad t>0 .
$$

The inner and outer problems are found by determining the model equations valid in the appropriate regions, in the limit as $\epsilon$ approaches zero. The physical effect of $\epsilon$ approaching zero $(E \rightarrow \infty)$ is that the temperature band about the reference temperature for which the Arrhenius term is significant becomes very thin. Thus, the reaction behaves as a point heat source at the interface of the inert and active regions. For small times we pose the following reduced outer problem,

$$
\frac{\partial U_{I}^{(1)}}{\partial t}=\frac{\partial^{2} U_{I}^{(1)}}{\partial x^{2}}, \quad 0<x<\lambda \text { and } \frac{\partial U_{A}^{(1)}}{\partial t}=\frac{\partial^{2} U_{A}^{(1)}}{\partial x^{2}}, \quad x>\lambda,
$$

with initial and boundary conditions $(2.5,2.6)$. The nonlinear term in Eq. (2.4b) and transition condition (2.7b) are removed and in their absence an appropriate jump condition is added

$$
\left.\left[\frac{\partial U^{(1)}(x, t)}{\partial x}\right]\right|_{x=\lambda^{-}} ^{x=\lambda^{+}}=g\left(U^{(1)}(\lambda, t)\right) .
$$

The form of $g$ will be determined by an inner layer analysis.

Inner layer analysis. The associated transition layer system is found by expanding the spatial variable, $x$, on the reactive side of the interface point, $x=\lambda$. Within this region we approximate the temperature perturbation by $U_{A}^{(1)}(\xi, t)=u_{1}(\xi, t)+$ $\epsilon u_{2}(\xi, t)$ where $\xi=\frac{1}{\epsilon}(x-\lambda), \quad 0<\xi<\infty$. Substitution into (2.4b) and applying matching conditions we find, upon equating terms of equal orders in $\epsilon$,

$$
\frac{d^{2} u_{1}}{d \xi^{2}}=0
$$


and

$$
\frac{d^{2} u_{2}}{d \xi^{2}}=-\exp \left[u_{1}(\xi, t)-\frac{1}{\epsilon} \theta(\xi, t ; \epsilon, \lambda)\right]
$$

where

$$
\theta(\xi, t ; \epsilon, \lambda) \equiv \operatorname{erf}\left(\frac{\epsilon \xi}{2 \sqrt{t}}\right)+\operatorname{erfc}\left(\frac{\epsilon \xi}{2 \sqrt{t}}+\frac{\lambda}{\sqrt{t}}\right) .
$$

The magnitude of $\theta$ dictates the behavior of the system. The order of $\theta$ depends on both $\epsilon$ and $\lambda$. Thus, a complete analysis consists of calculating a critical $\lambda$ (delineating sub from super-critical behavior) for each given small $\epsilon$.

Inner and outer solutions. Solutions to the outer problem can be written in the form of a Green's solution involving the unknown heat flux at the interface $x=\lambda$. The solution of $(2.8,2.5,2.6)$ is

$$
\begin{aligned}
U_{I}^{(1)}(x, t)= & \left.\frac{1}{\sqrt{\pi}} \int_{0}^{t} \frac{1}{\sqrt{t-s}}\left[\frac{\partial U_{I}^{(1)}(x, t)}{\partial x}\right]\right|_{x=\lambda^{-}} \\
& \cdot\left[\exp \left[-(x-\lambda)^{2} / 4(t-s)\right]+2 \sum_{n=1}^{\infty} \exp \left[-[x+\lambda(2 n-1)]^{2} / 4(t-s)\right]\right] d s, \\
& 0<x<\lambda, \quad t>0,
\end{aligned}
$$

for the inert region, while for the active region we have

$$
\begin{aligned}
& U_{A}^{(1)}(x, t)=-\left.\frac{1}{\sqrt{\pi}} \int_{0}^{t} \frac{1}{\sqrt{t-s}}\left[\frac{\partial U_{A}^{(1)}(x, t)}{\partial x}\right]\right|_{x=\lambda^{+}} \exp \left[-(x-\lambda)^{2} / 4(t-s)\right] d s . \\
& \lambda<x<\infty, \quad t>0 .
\end{aligned}
$$

We now proceed to construct the solutions of the inner problem $(2.10-2.11)$. The first step is to integrate (2.10) twice and apply matching conditions. One finds that $u_{1}(\xi, t)$ is a function of time alone, namely $u_{1}(\xi, t)=u(t)$. Next integrate (2.11) once to get

$$
\left.\frac{\partial u_{2}(\xi, t)}{\partial \xi}\right|_{\xi=\infty}-\left.\frac{\partial u_{2}(\xi, t)}{\partial \xi}\right|_{\xi=0}=-e^{u(t)} F(t),
$$

where $F(t)=\int_{0}^{\infty} \exp \left[\frac{-1}{\epsilon} \theta(\xi, t ; \epsilon, \lambda)\right] d \xi$. The evaluation of $F(t)$, for $\epsilon$ small, can be accomplished by using Laplace's method [3] which results in $F(t)=$ $\sqrt{\pi t} \exp \left[-\frac{1}{\varepsilon} \operatorname{erfc}\left(\frac{\lambda}{\sqrt{t}}\right)\right] /\left(1-\exp \left(-\lambda^{2} / t\right)\right)$.

Matching process. The matching process is accomplished by using the inner and outer solutions and matching conditions to derive an equation for the surface temperature perturbation, $u(t)$. When the outer solutions, (2.12 and 2.13), are evaluated at the interface $x=\lambda^{ \pm}$, we can write

$$
\begin{array}{r}
u(t)=U_{I}^{(1)}\left(\lambda^{-}, t\right)=\left.K_{0}\left[\frac{\partial U_{I}^{(1)}(x, t)}{\partial x}\right]\right|_{x=\lambda^{-}}+\left.2 \sum_{n=1}^{\infty} K_{n}\left[\frac{\partial U_{I}^{(1)}(x, t)}{\partial x}\right]\right|_{\left.\right|_{x=\lambda^{-}}} \\
0 \leq t
\end{array}
$$


and

$$
u(t)=U_{A}^{(1)}\left(\lambda^{+}, t\right)=-\left.K_{0}\left[\frac{\partial U_{A}^{(1)}(x, t)}{\partial x}\right]\right|_{x=\lambda^{+}}, \quad 0 \leq t
$$

where

$$
K_{n}[f(t)] \equiv \frac{1}{\sqrt{\pi}} \int_{0}^{t} \frac{1}{\sqrt{t-s}} f(s) \exp \left[-\frac{\lambda^{2} n^{2}}{(t-s)}\right] d s, \quad 0 \leq t, n=0,1,2, \ldots
$$

If $\lambda$ is large, $K_{n}$, for $n \geq 1$, can be neglected. This condition will be justified analytically in Sec. 3 and numerically confirmed in Sec. 4. In this case, the sum of (2.15) and (2.16) can be written as

$$
2 u(t)=\frac{1}{\sqrt{\pi}} \int_{0}^{t} \frac{1}{\sqrt{t-s}}\left[\frac{\partial U_{I}^{(1)}\left(\lambda^{-}, t\right)}{\partial x}-\frac{\partial U_{A}^{(1)}\left(\lambda^{+}, t\right)}{\partial x}\right] d s
$$

By the matching conditions we have,

$$
\left[\frac{\partial U_{A}^{(1)}\left(\lambda^{+}, t\right)}{\partial x}-\frac{\partial U_{I}^{(1)}\left(\lambda^{-}, t\right)}{\partial x}\right]=\left[\frac{\partial u_{2}(\infty, t)}{\partial \xi}-\frac{\partial u_{2}(0, t)}{\partial \xi}\right] .
$$

Thus, by (2.14), (2.17), and (2.18) we have,

$$
u(t)=\frac{1}{2} K_{0}\left[\sqrt{\pi t} \exp \left[u(t)-\frac{1}{\epsilon} \operatorname{erfc}\left(\frac{\lambda}{\sqrt{t}}\right)\right] /\left(1-\exp \left(\frac{-\lambda^{2}}{t}\right)\right)\right] .
$$

The comparison theory used in the next chapter leads to bounds on a 'critical' curve $\left(\epsilon, \lambda_{c}(\epsilon)\right)$ which separates the super critical (iginition) region $\lambda>\lambda_{c}(\epsilon)$, from the subcritical $\lambda<\lambda_{c}(\epsilon)$, region. We show that for $\epsilon$ small a lower bound on $\lambda_{c}$ is large. Thus with some confidence we can neglect terms involving $K_{n}$ when discussing the situations in which the system is close to or at criticality.

3. Critical behavior. The application of A.E.A. to (1.1) has reduced the determination of criticality from the study of the behavior of the solution of a nonlinear partial differential equation to the study of the solution of the Volterra integral equation (2.19) rewritten as,

$$
u(t)=\frac{1}{2} \int_{0}^{t}\left[\sqrt{\frac{s}{t-s}}\left(\exp \left[u(s)-\frac{1}{\epsilon} \operatorname{erfc}\left(\frac{\lambda}{\sqrt{s}}\right)\right]\right)\left(1-\exp \left(-\frac{\lambda^{2}}{s}\right)\right)^{-1}\right] d s
$$

The solution of (3.1) represents the perturbation from the inert temperature profile at the interface. The magnitude and duration of the effects of heat conduction and reaction heat release mechanisms on the solution $u(t)$ depend on the two parameters $\lambda$, the scaled hot slab size, and $\varepsilon^{-1}$ the Zeldovitch number. For a given $\varepsilon$, and small enough $\lambda$, hot slab ignition is thwarted by conduction effects. For the same value of $\epsilon$ and large enough values of $\lambda$ the heat reservoir in the hot slab contains enough heat energy to maintain a high interface temperature and ignition occurs. The behavior of the solution of (3.1) reflects these two cases and is delineated by a sudden vast change in time scale as the system moves from the sub to super-critical regime. Should the 
latter case be true, the system is said to be critical and the solution $u(t)$ blows up in a short time frame:

$$
u(t) \rightarrow \infty \text { as } t \rightarrow t_{B}^{-} \approx O(1),
$$

where $t_{B}$ represents the blow up time of the solution. The former case is noncritical. Although the solution of (3.1) always blows up in finite time, the noncritical case results in a blow up time which is very large. In this section we use comparison theorems to construct upper and lower solutions of (3.1). These solutions, like $u(t)$, are dependent on $\epsilon$ and $\lambda$ and are used to determine bounds on the critical region in $\varepsilon, \lambda$ parameter space.

Comparison theory. The existence of a maximally defined unique solution of (3.1) is ensured by standard results of nonlinear integral equation theory (see Miller [10]). We employ comparison theory to construct upper and lower solutions that bound the solution. The comparison theorem is based on the observation that if integrands in the two equations

$$
w(t)=\int_{0}^{t} G(t, s, w(s)) d s
$$

and

$$
u(t)=\int_{0}^{t} g(t, s, u(s)) d s
$$

satisfy the condition

$$
G(t, s, v) \geq g(t, s, v) \text { in } R=\left\{0 \leq s \leq t<t^{*} ; 0 \leq v<\infty\right\},
$$

then any solution $u(t)$ of (3.3) will satisfy

$$
u(t) \leq \int_{0}^{t} G(t, s, u(s)) d s, \quad 0 \leq t \leq t^{*}
$$

Here $t^{*}$ is the maximal time of existence of the solution of (3.2). Thus, given any solution $w(t)$ of $(3.2)$ on $\left[0, t^{*}\right)$ the result, $u(t) \leq w(t)$ on $\left[0, t^{*}\right)$ follows directly. The statement and proof of the theorem is found in Miller.

We construct upper and lower solutions of (3.1) by defining appropriate upper and lower comparison equations which, when compared to (3.1), satisfy the stated hypotheses of the theorem. The lower solution we construct blows up in finite time, thus the solution of (3.1) must also. If their respective blow up times are denoted $t_{B L}$ and $t_{B}$, then $t_{B} \leq t_{B L}$ and $t^{*} \equiv t_{B}$. The upper solutions we construct do not blow up in finite time. Nevertheless, the upper limit of their validity $t^{*}$, bounds $t_{B}$ from below giving $t^{*} \equiv t_{U} \leq t_{B} \leq t_{B L}$.

Lower solutions. An appropriate comparison equation for the construction of the lower solution is

$$
v(t)=\delta \int_{0}^{t} \gamma(s) e^{v(s)} d s, \quad 0 \leq t<t^{*}<\infty,
$$

where

$$
\gamma(s)= \begin{cases}0, & 0 \leq s \leq \sigma \\ \gamma, & \sigma<s \leq t\end{cases}
$$


Here $\sigma, \delta$, and $\gamma$ will be choosen so that $\delta$ bounds $g(s ; \lambda, \epsilon) \equiv \frac{1}{2} \exp \left[-\frac{1}{\varepsilon} \operatorname{erfc}(\lambda / \sqrt{s})\right]$ from below and $\gamma(s)$ bounds $h(t, s) \equiv \sqrt{s /(t-s)}$ from below. The solution of (3.4) is

$$
v(t)= \begin{cases}0, & 0 \leq t<\sigma \\ -\ln [1-\gamma \delta(t-\sigma)], & \sigma \leq t \leq t_{B L},\end{cases}
$$

where $t_{B L}$ is the time of blow-up for $v(t)$. By observation, $t_{B L}=\frac{1}{\delta \gamma}+\sigma$. Since $1<\left(1-\exp \left(-\lambda^{2} / s\right)\right)^{-1}, \lambda>0, s>0, v(t)$ is a lower solution of $u(t)$, thus $u(t)$ must blow up at a time $t_{B}<t_{B L}$. Hence, the interval for which the comparison theorem is valid is $\left[0, t^{*}\right), t^{*} \equiv t_{B}<t_{B L}$.

In order to accomplish the construction of $v(t)$, we assume for the moment that $\delta$ is known. The function $h(t, s)$ satisfies $h\left(t_{B L}, t_{B L} / a\right) \leq h(t, s)$ for $\sigma \leq s \leq t \leq t_{B L}$ if $\sigma \equiv t_{B L} / a$ and $a>1$. Consequently, $\gamma \equiv h\left(t_{B L}, t_{B L} / a\right)=(a-1)^{-1 / 2}$ is a valid lower bound of $h(t, s)$. The lower solution becomes

$$
v(t)= \begin{cases}0, & 0 \leq t<t_{B L} / a \\ -\ln \left[1-\delta(a-1)^{-1 / 2}\left(t-t_{B L} / a\right)\right], & t_{B L} / a \leq t<t_{B L},\end{cases}
$$

where $t_{B L}=a / \delta \sqrt{a-1}$.

We now find the lower solution with smallest blow up time $t_{B L}$ for given $\lambda, \epsilon$, and $\delta$. This is done by minimizing $t_{B L}$ with respect to $a$, subject to $a \geq 1$. This gives $a_{\min }=2$. In summary, we have a lower solution $v(t)$ defined by (3.6) with $a=2, \sigma=\delta^{-1}$, and $t_{B L}=2 \delta^{-1}$ giving

$$
v(t)= \begin{cases}0, & 0 \leq t \leq \delta^{-1} \\ -\ln (2-\delta t), & \delta^{-1}<t<2 \delta^{-1}\end{cases}
$$

Finally, we determine the value of $\delta$ which gives the lower solution with smallest blow up time for given $\lambda$ and $\epsilon$. Since $v(t)=0$ on $0 \leq s \leq t \leq \sigma \equiv \delta^{-1}$, the lower solution with least blow up time will result if we use

$$
\delta=\min _{\delta^{-1} \leq s \leq 2 \delta^{-1}} g(s ; \lambda, \epsilon) .
$$

Since $g$ is strictly decreasing with respect to $s$, we replace (3.7) by

$$
\delta=g\left(2 \delta^{-1} ; \lambda, \epsilon\right)=\frac{1}{2} \exp \left[-\frac{1}{\varepsilon} \operatorname{erfc}\left(\lambda \sqrt{\frac{\delta}{2}}\right)\right],
$$

a fixed point problem for $\delta$. The fixed points of (3.8) are dependent on the values of $\lambda$ and $\epsilon$. We find them by introducing the trasformation $\omega=\left(\lambda^{2} / 2\right) \delta$ which gives

$$
\frac{2}{\lambda^{2}} \omega=\triangle(\omega) \equiv \frac{1}{2} \exp \left[-\frac{1}{\epsilon} \operatorname{erfc}(\sqrt{\omega})\right] .
$$

The graph of $\triangle(\omega)$ is shown in Fig. 3.1 along with the graphs of $f(\omega ; \lambda)=\left(2 / \lambda^{2}\right) \omega$ for several values of $\lambda$. The existence of two points of tangency as depicted in Fig. 3.1 only arises for values of $\epsilon<\epsilon^{*}$, where $\epsilon^{*}$ is defined below. This can be seen by 


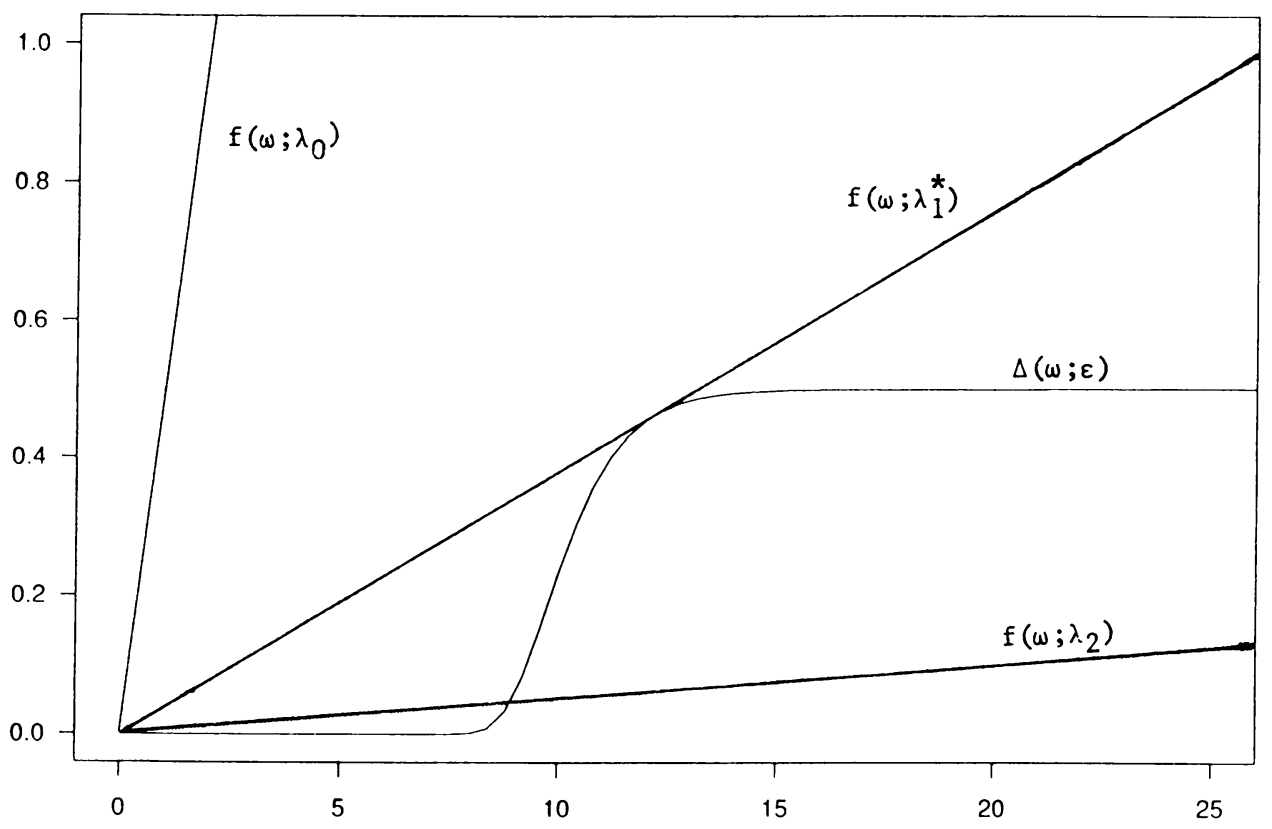

FIG. 3.1. Solutions of the fixed point problem (3.10a) for small $\left(\lambda_{0}\right)$, critical $\left(\lambda_{1}^{*}\right)$, and large $\left(\lambda_{2}\right)$ values of $\lambda$.

imposing the conditions needed for the existence of two points of tangency denoted $\left(\omega_{i}, \lambda_{i}\right), i=1,2$

$$
2 \frac{\omega_{i}}{\lambda_{i}^{2}}=\triangle\left(\omega_{i}\right)
$$

and

$$
\frac{2}{\lambda_{i}^{2}}=\triangle^{\prime}\left(\omega_{i}\right)=\frac{1}{\epsilon \sqrt{\pi}} e^{-\omega_{i}} \omega_{i}^{-1 / 2} \triangle\left(\omega_{i}\right)
$$

Substitution of (3.10a) into (3.10b) gives $\epsilon \sqrt{\pi}=\omega_{i}^{1 / 2} e^{\omega_{i}}$ which has no solutions for $\epsilon>\epsilon^{*} \equiv(2 \pi e)^{-1 / 2}$, one for $\epsilon=\epsilon^{*}$ and two when $\epsilon<\epsilon^{*}$ (the situation depicted in Fig. 3.1). The use of the transformation $\omega=\left(\lambda^{2} / 2\right) \delta$, in $t_{B L}=2 \delta^{-1}$ results in

$$
t_{B L}=\frac{\lambda^{2}}{\omega(\lambda, \epsilon)},
$$

where $\omega(\lambda, \epsilon)$ is used to represent any fixed point of (3.9). It is evident that the second point of tangency will correspond to the lower solution with smallest blow up time. Thus, the super-critical curve $\left(\epsilon, \lambda_{\text {sup }}(\epsilon)\right)$, above which there exists lower solutions which blow up in small time, can be found by determining $\omega_{1}(\epsilon)$ and $\lambda_{1}(\epsilon)$. It is for these values of $\lambda$ that a lower solution with small blow up time exists. We set $\lambda_{\text {sup }}(\epsilon) \equiv \lambda_{1}(\epsilon)$. An asymptotic expression for $\lambda_{\text {sup }}(\epsilon)$ can be derived for small $\epsilon$. In this case, $\omega_{1}$ must be large giving $\epsilon \sqrt{\pi} \simeq e^{-\omega_{1}}$. The solution $\omega_{1}$, when plugged into (3.10a), gives an asymptotic expression for $\lambda_{\text {sup }}$,

$$
\lambda_{\text {sup }}^{2}(\varepsilon)=4|\ln (\varepsilon \sqrt{\pi})| \exp \left[\frac{1}{\varepsilon} \operatorname{erfc}\left(|\ln (\varepsilon \sqrt{\pi})|^{1 / 2}\right)\right](1+o(1)) .
$$


Upper solutions. We now construct an upper solution of $u(t)$. Although this upper solution does not blow up at a finite value of time the interval over which it is valid will indicate whether the solution to Eq. (3.1) blows up in a short interval of time, or not.

To begin, we propose an upper equation of the form

$$
\omega(t)=\int_{0}^{t} \sqrt{\frac{s}{t-s}} \cdot \delta(s) e^{m} d s, \quad 0 \leq t \leq t^{*},
$$

where $m$ and $t^{*}$ are to be determined and $\delta(s)$ will be defined for given $\lambda$ and $\epsilon$. The form of the equation has been chosen to best reflect the behavior of the original equation, while at the same time allowing an explicit determination of $\delta, m$, and $t^{*}$. The goal of this section is to find $t^{*}$ and maximize it with respect to $m$. To do this we assume $\delta(s)$ is known. We let $h(t, s)=\sqrt{\frac{s}{t-s}}$ and $g(s ; \lambda, \varepsilon)=\frac{1}{2} \exp \left[-\frac{1}{\varepsilon} \operatorname{erfc}(\lambda / \sqrt{s})\right] /\left(1-\exp \left(-\lambda^{2} / s\right)\right)$. Define $\delta(s)$ as

$$
\delta(s)= \begin{cases}\delta_{1}, & 0 \leq s \leq s_{1} \\ \delta_{2}, & s_{1}<s \leq s_{2}\end{cases}
$$

where $\delta_{1}$, and $\delta_{2}$, are defined by $\delta_{1} \equiv g\left(s^{*} ; \lambda, \epsilon\right)$ with $g^{\prime}\left(s^{*} ; \lambda, \epsilon\right)=0$ and $g^{\prime \prime}\left(s^{*} ; \lambda, \epsilon\right)<0, \delta_{2} \equiv \epsilon \delta_{1}$ and $s_{1}$ and $s_{2}$ are found by solving $\delta_{2}=g\left(s_{1} ; \lambda, \epsilon\right)=$ $g\left(s_{2} ; \lambda, \epsilon\right) . \omega(t)$ is strictly increasing in $t$ therefore if $\omega\left(t^{*}\right)=m$, then $\omega(t)<m$, for $0 \leq t \leq t^{*}$. Obviously, $e^{m} \geq e^{\omega(s)}, 0 \leq s \leq t^{*}$ and since $\delta(s) \geq g(s ; \lambda, \epsilon)$ on $\left[0, s_{2}\right]$, we know that $\omega(t) \geq u(t)$ for $0 \leq t \leq t^{*}$ as long as $t^{*} \leq s_{2}$, by the comparison theorem. The values for $t^{*}$ and $m$ are determined by considering two cases.

Case I. $\left[t^{*} \leq s_{1}\right]$ : In this case $\omega(t)=\delta_{1} e^{m} \int_{0}^{t} \sqrt{\frac{s}{t-s}} d s$, for $0 \leq t \leq t^{*}$. The integral part of $\omega(t)$ can be evaluated by introducing the transformation $\eta=\frac{s}{t}$ which results in $\int_{0}^{t} \sqrt{\frac{s}{t-s}} d s=t B\left(\frac{1}{2}, \frac{3}{2}\right)$. Where $B(p, q)$ is the Beta function with value $B(p, q)=\frac{\Gamma(p) \Gamma(q)}{\Gamma(p+q)}$ [1]. Thus, we have $\omega(t)=\delta_{1} e^{m} \frac{\pi}{2} t$, for $0 \leq t \leq t^{*}$. In order to determine $t^{*}$ and $m$, we impose the condition $\omega\left(t^{*}\right)=m$ which gives $t^{*}=\left(2 / \pi \delta_{1}\right) m e^{-m}$. When $m=1, t^{*}$ is maximized. This gives $t^{*}=\left(2 / \pi e \delta_{1}\right) \equiv t_{1}$ and the upper solution is $\omega(t)=t / t_{1}$, for $0 \leq t \leq t_{1}$.

Case II. [ $\left.s_{1} \leq t^{*}\right]$ : In this case $w(t)$ is given by,

$$
w(t)= \begin{cases}\delta_{1} e^{m} \int_{0}^{t} \sqrt{\frac{s}{t-s}} d s & \text { for } 0 \leq t \leq s_{1} \\ \delta_{1} e^{m} \int_{0}^{s_{1}} \sqrt{\frac{s}{t-s}} d s+\delta_{2} e^{m} \int_{s_{1}}^{t} \sqrt{\frac{s}{t-s}} d s & \text { for } s_{1} \leq t \leq t^{*}\end{cases}
$$

Since $\omega(t)$ cannot be evaluated in a way that can be effectively used in the analysis that follows, it is prudent to define a new function $\hat{\omega}(t)$. This function is an upper bound of $\omega(t)$, and therefore of $u(t) . \hat{\omega}(t)$ has an explicit representation and can be better put to use in the analysis that follows.

By inspection,

$$
\int_{0}^{s_{1}} \sqrt{\frac{s}{s_{1}-s}} d s \geq \int_{0}^{s_{1}} \sqrt{\frac{s}{t-s}} d s, \quad 0 \leq s_{1} \leq t
$$


and

$$
\int_{0}^{t} \sqrt{\frac{s}{t-s}} d s \geq \int_{s_{1}}^{t} \sqrt{\frac{s}{t-s}} d s, \quad 0 \leq s_{1} .
$$

We define $\hat{\omega}(t)$ by

$$
\hat{w}(t)= \begin{cases}\delta_{1} e^{m} \int_{0}^{t} \sqrt{\frac{s}{t-s}} d s & \text { for } 0 \leq t \leq s_{1} \\ \delta_{1} e^{m} \int_{0}^{s_{1}} \sqrt{\frac{s}{s_{1}-s}} d s+\delta_{2} e^{m} \int_{0}^{t} \sqrt{\frac{s}{t-s}} d s & \text { for } s_{1}<t \leq t^{*}\end{cases}
$$

Evaluating the integrals gives

$$
\hat{\omega}(t)= \begin{cases}\delta_{1} e^{m} \pi t / 2, & 0 \leq t \leq s_{1} \\ \delta_{1} e^{m} \pi s_{1} / 2+\delta_{2} e^{m} \pi t / 2, & s_{1}<t \leq t^{*} .\end{cases}
$$

Since we have assumed that $s_{1}<t^{*}$, we find as in Case I, the maximum of $t^{*}$ with respect to $m$ occurs at $m=1$, which gives

$$
t^{*}=\frac{1}{\delta_{2}}\left(\frac{2}{\pi e}-\delta_{1} s_{1}\right) \equiv t_{2} .
$$

With $t^{*}$ and $m$ known we complete the construction of $\hat{\omega}(t)$ by finding $s_{1}, s_{2}, \delta_{1}$, and $\delta_{2}$ when $\lambda$ and $\epsilon$ are given. Define $s^{*}$ to be the value of $s$ for which $g(s ; \lambda, \epsilon)$ attains its local maximum. Then $\delta_{1} \equiv g\left(s^{*} ; \lambda, \epsilon\right)$. For small $\epsilon, s^{*} \approx$ $\pi \epsilon^{2} \lambda^{2}(1+O(\epsilon))$ which gives, $\delta_{1} \approx 1$. Thus, $\delta_{2} \approx \varepsilon$ and $s_{1}$ and $s_{2}$ are found by solving $\delta_{2}=g\left(s_{1} ; \lambda, \varepsilon\right)=g\left(s_{2} ; \lambda, \varepsilon\right), s_{1}<s_{2}$. Let $\omega_{i}=\lambda / \sqrt{s_{i}}$ then

$$
\delta_{2} \equiv \frac{1}{2}\left(1-\exp \left(-\omega_{i}^{2}\right)\right)^{-1} \exp \left[-\frac{1}{\epsilon} \operatorname{erfc}\left(\omega_{i}\right)\right], \quad i=1,2 .
$$

When $\epsilon$ is small $\omega_{2}$ is small and is determined asymptotically as the solution of

$$
\epsilon=\frac{1}{2}\left(1-\exp \left(-\omega_{2}^{2}\right)\right)^{-1} \exp \left(-\frac{1}{\epsilon}\right)
$$

yielding

$$
\omega_{2} \approx \frac{1}{\sqrt{2 \varepsilon}} \exp \left(\frac{-1}{2 \epsilon}\right)<<1
$$

thus

$$
s_{2}=2 \lambda^{2} \epsilon \exp \left(\frac{1}{\epsilon}\right)>>1 \text {. }
$$

In a similar way, when $\epsilon$ is small, (3.15) yields an asymptotic expression for $\omega_{1}$ giving

$$
\omega_{1} \approx|\ln (\epsilon \sqrt{\pi}|\ln (\epsilon)|)|^{1 / 2}
$$

resulting in

$$
s_{1} \approx \frac{\lambda^{2}}{|\ln (\epsilon \sqrt{\pi}|\ln (\epsilon)|)|} .
$$

With this information the conditions for criticality can be imposed yielding the desired result. Application of the criterion for Case I, $t_{1} \leq s_{1}$, gives

$$
\frac{2}{\pi e} \leq \frac{\lambda^{2}}{|\ln (\epsilon \sqrt{\pi}|\ln (\epsilon)|)|}
$$


or

$$
\lambda^{2} \geq \frac{2}{\pi e}|\ln (\epsilon \sqrt{\pi}|\ln (\epsilon)|)|
$$

Case II's criterion is the opposite inequality. Thus we can define a sub-critical curve

$$
\lambda_{\text {sub }}^{2}(\epsilon)=\frac{2}{\pi e}|\ln (\epsilon \sqrt{\pi}|\ln (\epsilon)|)|
$$

which delineates the region $\lambda>\lambda_{\text {sub }}$ in which upper solutions are valid for only a short time $t_{1}$, from the region $\lambda<\lambda_{\text {sub }}$ in which upper solutions are valid over long times $t_{2}$ indicating that ignition does not take place.

Results of the comparison theory analysis. Two curves have been defined in $\epsilon-\lambda$ space. The first, $\lambda_{\text {sup }}(\epsilon)$, delineates a region $\lambda \geq \lambda_{\text {sup }}$ in which lower solutions $v(t)$, of Eq. (3.1) blow up in small finite time. Since $u(t)$, the solution of $(3.1)$, is bounded below by $v(t)$, then $u(t)$ must also blow up in small finite time. In this case we say the system is super-critical and predict that ignition will take place. The second curve, $\lambda_{\text {sub }}(\epsilon)$, distinguishes two regions as well. For $\lambda<\lambda_{\text {sub }}$, the upper solution is valid over a long time interval given by (3.14). Since the interval of validity bounds from below the blow up time $t_{B}$, of $u(t)$, within this region the perturbation cannot blow up within moderate times. In this case we say the system is sub-critical and predict that ignition will not take place. The large values of $\lambda_{\text {sub }}$, for $\epsilon$ small, lend confidence to the working hypothesis of Sec. 2 that $K_{n}$ can be neglected.

The region between the two curves is a transition region which this asymptotic analysis does not clearly distinguish. In the next section a numerical technique is used to verify the results presented here.

4. Numerical study of the Volterra equation. Although the analysis of the previous chapter has led to an estimate of the desired results, the numerical solution of (3.1) gives confidence and support to those results. The behavior of solutions in the transition region $\lambda_{U}(\epsilon)<\lambda<\lambda_{L}(\epsilon)$, are of particular interest as the results obtained so far are not able to address that region.

Numerous integration techniques with high-order accuracy are available to compute solutions of nonlinear Volterra equations whose kernel is well-behaved [9]. When the kernel, or one of its derivatives, is unbounded these techniques must be modified to account for the singularity. An algorithm known as the product integration method (P.I.M.) is one such suitable technique. Although it is used to solve singular equations, its accuracy, when applied to certain classes of singularities, compares with the accuracy of standard methods used on nonsingular equations with approximately the same amount of computer work. An unexpected advantage is P.I.M.'s ability to distinguish blow up behavior. Very little analysis appears in the literature concerning P.I.M. but a development of standard accuracy results is developed in Linz [9], while related convergence results are presented by Kershaw [4].

The implementation of P.I.M. for (3.1) proceeds as follows. Using definitions $h(t, s) \equiv \sqrt{s / t-s}$ and $g(s, u(s)) \equiv \frac{1}{2} \exp \left[u(s)-\frac{1}{\varepsilon} \operatorname{erfc}(\lambda / \sqrt{s})\right] /\left(1-\exp \left(-\lambda^{2} / s\right)\right)$ gives,

$$
u(t)=\int_{0}^{t} h(t, s) g(s, u(s)) d s
$$


Here $h$ contains the singular part of the kernel while $g$ is the well-behaved factor. A fixed time step of length $\Delta t>0$, is chosen with $t_{n}=n \Delta t, \quad n=0,1,2, \ldots, N$, with $t_{0}=0$ and $t_{N} \simeq t$. This discretization yields the following approximation of (4.1):

$$
u\left(t_{N}\right)=\sum_{n=0}^{N-1} \int_{t_{n}}^{t_{n+1}} h\left(t_{N}, s\right) g(s, u(s)) d s
$$

An interpolating function is used to approximate the smooth factor $g$. The order of convergence is essentially dependent on the order of accuracy of the interpolating function. Within each subinterval $\left[t_{n}, t_{n+1}\right]$, the linear Lagrange interpolating formula is used to give $O\left(h^{2}\right)$ accuracy. If we define $g_{n}=g\left(t_{n}, u\left(t_{n}\right)\right)$ then (4.2) is replaced by

$$
u_{N}=\frac{1}{h} \sum_{n=0}^{N-1}\left[g_{n} \int_{t_{n}}^{t_{n+1}} h\left(t_{n}, s\right)\left(t_{n+1}-s\right) d s+g_{n+1} \int_{t_{n}}^{t_{n+1}} h\left(t_{N}, s\right)\left(s-t_{n}\right) d s\right],
$$

where $u_{N}$ is the computed approximation to $u\left(t_{N}\right)$. In this case, closed form expressions for the integrals exist. The result can be used to give an equation for $u_{N}$ of the form

$$
u_{N}=A_{N}+B_{N} \cdot g_{N}, \quad N=1,2,3 \ldots .
$$

Here $A_{N}$ represents the sum of the known terms and $B_{N}$ the coefficient of the factor involving the unknown $u_{N}$. Since the form of the nonlinearity in $g$ is $e^{u}$, (4.4) is a transcendental equation that can be solved using standard root-finding routines. In order to determine whether a parameter pair $(\epsilon, \lambda)$ is critical or not, we must compute $u_{N} \approx u\left(t_{N}\right)$ until $u_{N}$ exhibits behavior analogous to blow up in finite time of the solution $u(t)$, of (4.1). Of course computation of the blow up in finite time of a solution is not possible. Instead we ask, what behavior of this computed approximation corresponds to blow up in finite time of $u(t)$ ? This question can be answered, at least partially, by considering the solutions of (4.4). Let,

$$
F_{N}\left(u_{N}\right)=A_{N}+B_{N}^{\prime} e^{u_{N}}-u_{N},
$$

where $B_{N}^{\prime}$ is the product of $B_{N}$ with the factors of $g_{N}$ other than $e^{u_{N}}$. It can be shown that $A_{N}>0$ and that $B_{N}^{\prime}>0$. Under these conditions, $F_{N}\left(u_{N}\right)$ has zero, one or two solutions depending on whether $F_{N}\left(u_{N}^{\star}\right)=A_{N}+1+\ln B_{N}$ is greater than, equal to, or less than zero, respectively. Here, $u_{N}^{\star}=-\ln B_{N}$ is the global minimum of $F_{N}\left(u_{N}\right)$. Should two solutions exist (always true for $N$ small), the smaller is the desired solution while the larger is an artifact of the discretization and interpolation. It is clear that $F_{N}\left(u_{N}^{\star}\right)=0$ when $B_{N}=\exp \left(-A_{N}+1\right)$. Thus, there are two solutions for $B_{N}$ less than, and no solutions for $B_{N}$ greater than, this value. We conjecture that the blow up in finite time of the solution of (4.1) is reflected in the numerical solution by the lift-off from the $u_{N}$ axis of the graph of the function $F_{N}\left(u_{N}\right)$, i.e., at the value of $N$ for which $F_{N}\left(u_{N}^{\star}\right)=0$. Since this is a discretization it is unlikely that this condition will actually be obtained. Rather we look for the value of $N$ such that $F_{N}\left(u_{N}^{\star}\right)<0$ and $F_{N+1}\left(u_{N+1}^{\star}\right)>0$. The computed blow up time can be defined as $t_{B_{N}}=N \triangle t$. 
TABLE 4.1. Numerical results for test problem (4.5).

\begin{tabular}{|c|c|c|c|c|}
\hline$t_{n}$ & $h=0.1$ & $h=0.01$ & $h=0.001$ & $u(t)=\ln (1-t)$ \\
\hline 0.1 & 0.09549 & 0.10525 & 0.10535 & 0.10536 \\
\hline 0.2 & 0.21233 & 0.22302 & 0.22314 & 0.22314 \\
\hline 0.3 & 0.34474 & 0.35654 & 0.35667 & 0.35667 \\
\hline 0.4 & 0.49755 & 0.51067 & 0.51082 & 0.51082 \\
\hline 0.5 & 0.67832 & 0.69298 & 0.69314 & 0.69314 \\
\hline 0.6 & 0.89980 & 0.91610 & 0.91628 & 0.92619 \\
\hline 0.6 & 1.18654 & 1.20377 & 1.20397 & 1.20397 \\
\hline 0.8 & 1.59732 & 1.60927 & 1.60943 & 1.60943 \\
\hline 0.9 & 2.38998 & 2.30309 & 2.30259 & 2.30258 \\
\hline 0.99 & & 4.84834 & 4.60672 & 4.60517 \\
\hline 0.999 & & & 7.17108 & 6.90775 \\
\hline 1.000 & (undefined) & (undefined) & (undefined) & (undefined) \\
\hline
\end{tabular}

We applied P.I.M. to a Volterra equation which has an explicit solution and numerically verified the conjecture. Consider the equation,

$$
u(t)=\int_{0}^{t} e^{u(s)} d s, \quad t \geq 0,
$$

which has solution $u(t)=-\ln (1-t)$ and blow up time $t_{B}=1$. The resulting equation, when P.I.M. is applied to (4.5), is

$$
F_{N}\left(u_{N}\right)=A_{N}+B e^{u_{N}}-u_{N} \text {, }
$$

where

$$
A_{N}= \begin{cases}\frac{h}{2}, & N=1 \\ \frac{h}{2}\left[1+2 \sum_{n=1}^{N-1} e^{u_{n}}\right], & N=2,3,4, \ldots\end{cases}
$$

and $B=\frac{h}{2}$. In this case, $u_{N}^{\star}$ is independent of the time step $N$ :

$$
u_{N}^{\star} \equiv u^{\star}=-\ln \left(\frac{h}{2}\right) \text {. }
$$

When substituted into $F_{N}\left(u_{N}\right)$ this gives,

$$
F_{N}\left(u^{\star}\right)=A_{N}+1+\ln \frac{h}{2} \text {. }
$$




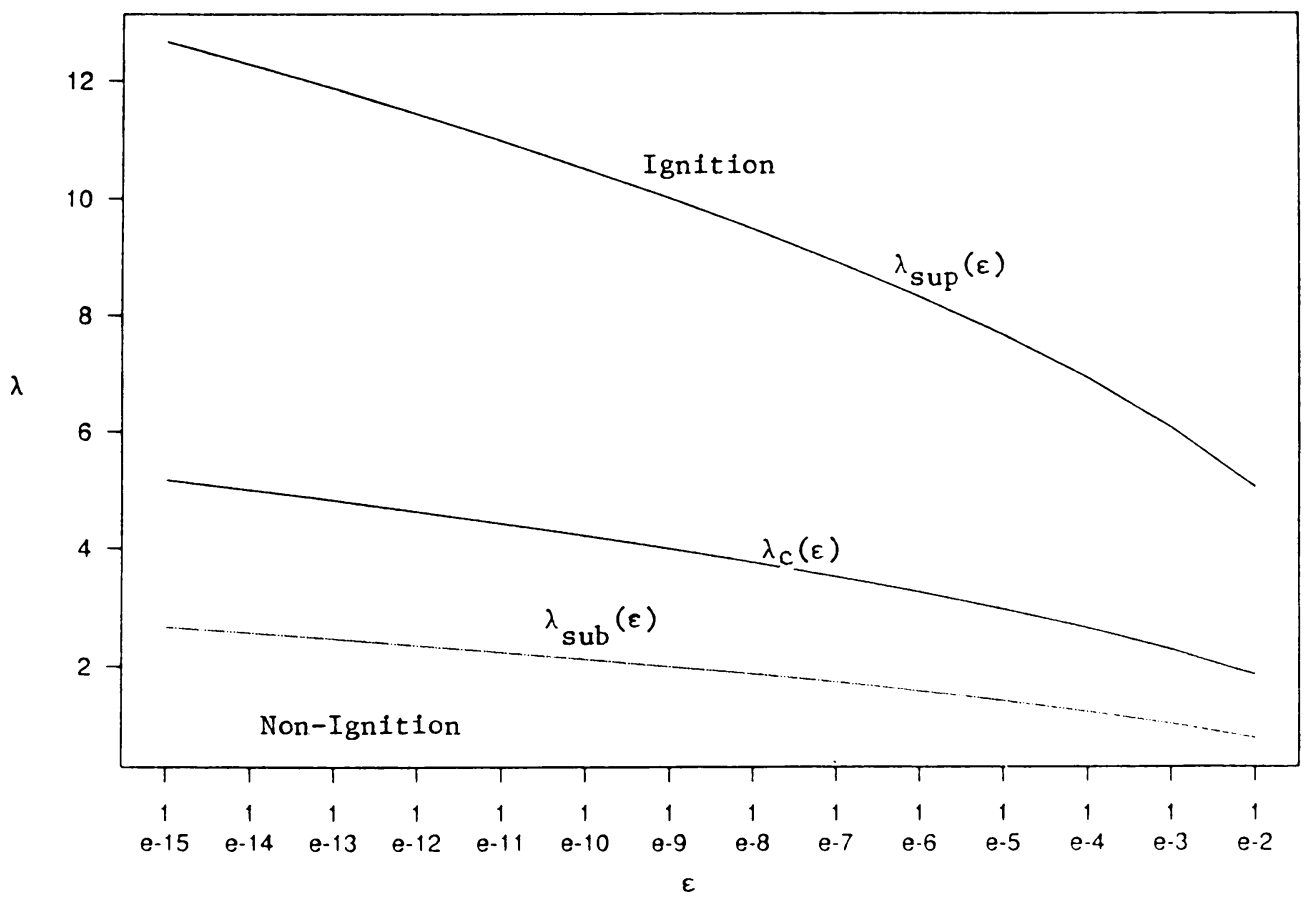

FIG. 4.1. Upper $\left(\lambda_{\text {sup }}(\varepsilon)\right)$ and lower $\left(\lambda_{\text {sub }}(\varepsilon)\right)$ bounds on computed critical curve $\left(\lambda_{c}(\varepsilon)\right)$.

Since $A_{N}$ is increasing with $N$ (see (4.6)), $F_{N}\left(u^{\star}\right)$ will become greater than zero at some point $t_{B_{N}}$. Note that $A_{1}=\frac{h}{2}$ thus $F_{N}\left(u_{N}^{\star}\right)<0$ for $h$ small enough. Results are shown in Table 4.1. The $O\left(h^{2}\right)$ accuracy is apparent. In addition, the blow up time is predicted within one time step for every $h$.

The results of the computation of $u_{N}$, the solution of (4.4), for values of $\epsilon$ and $\lambda$ in the transition region $\lambda_{\text {sub }}(\epsilon)<\lambda<\lambda_{\text {sup }}(\epsilon)$, indicate that there is a critical curve $\lambda_{c}(\epsilon)$, which is close to the lower bound $\lambda_{\text {sub }}(\epsilon)$. See Fig. 4.1. This is not surprising considering the approximations used in deriving the lower solutions. In particular, the definition of $\delta$ is a rather crude approximation and leads to a conservative upper bound of the critical curve. The numerical results indicate that the transition from sub-critical to super-critical is very sharp.

Conclusions. We have shown that critical conditions for hot slab ignition of an idealized reactive medium in one dimension can be determined based on hot slab size, initial temperatures and reaction rate parameters. Activation energy asymptotics is employed to reduce the determination of criticality from the behavior of the nonlinear partial differential equation (1.1) to the behavior of the solution of (3.1). The reduction is accomplished through a perturbation expansion of temperature coupled with a matching process near the interface of the inert and active mediums. The matching process results in an equation for the inner transition layer temperature perturbation that is shown to be spatially independent. Equation (3.1) is a nonlinear singular Volterra equation of the second kind. The factors in the kernel of (3.1) 
correspond to the mechanisms of interest; namely, heat conduction, hot slab heat loss, and reaction rate.

Although the integral equation's solution cannot be determined explicitly, a comparison principle is used to construct upper and lower solutions. These comparison solutions are then used to find bounds on the transition zone in $\lambda-\epsilon$ space where $\lambda$ is the scaled size of the hot spot and $\varepsilon^{-1}$ is the Zeídovich number. Analysis of the dependence of $t_{B L}$, the blow up time for the lower solution, on the two parameters $\lambda$ and $\epsilon$ leads to the determination of a curve $\lambda_{\text {sup }}(\epsilon)$ which defines the region $\left(\lambda>\lambda_{\text {sup }}(\epsilon)\right)$, in which lower solutions blow up in small finite time. Asymptotically, $\lambda_{\text {sup }}(\epsilon)$ has the form

$$
\lambda_{\text {sup }}^{2}(\epsilon)=4|\ln \epsilon \sqrt{\pi}| e^{(1 / \epsilon) \operatorname{erfc}\left(|\ln \epsilon \sqrt{\pi}|^{1 / 2}\right)} .
$$

In a similar way upper solutions are constructed and a related lower bound $\lambda_{\text {sub }}(\epsilon)$ is shown to exist. Its asymptotic form is

$$
\lambda_{\text {sub }}^{2}(\epsilon)=\frac{2}{\pi e} \mid \ln (\epsilon \sqrt{\pi}|\ln (\epsilon)|) .
$$

The blow up behavior cannot be distinguished in the transition region, $\lambda_{\text {sub }}(\epsilon)<\lambda<\lambda_{\text {sup }}(\epsilon)$, using the comparison theory. A numerical integration technique called the product integration method (P.I.M.) is used to find numerical bounds on a critical curve $\lambda_{c}(\epsilon)$.

It is possible that the analysis applied here can be extended to more complex modeling systems. One idea would be to combine the analysis of Liñan and Kindelan [8] with the results presented here to address complex two- and three-dimensional hot spot geometries. The key is to incorporate both local curvature effects and the size of the nonsymmetric hot spot. The first problem would be to determine an expression for the local inert temperature solution which takes into effect the heat loss from all of the boundary.

Acknowledgment. The author expresses his sincere appreciatation to Professor P.C. Fife of the University of Utah for numerous suggestions and encouragement throughout the course of this research.

\section{REFERENCES}

[1] R. C. Buck, Advanced Calculus, McGraw-Hill, New York, 1965

[2] H. S. Carslaw and J. C. Jaeger, Conduction of Heat in Solids, 2nd Ed., Oxford University Press, Oxford, 1959

[3] A. Erdelyi, Asymptotic Expansions, Dover, New York, 1956

[4] D. Kershaw, Some results for Abel-Volterra integral equations of the second kind, Treatment of Integral Equations by Numerical Methods, edited by C. T. H. Baker and G. F. Miller, Academic Press, London, 1982, pp. 273-282

[5] C. K. Law and H. K. Law, Flat-plate ignition with reactant consumption, Combustion Science and Technology 25, 1-8 (1981)

[6] A. Liñan and F. A. Williams, Theory of ignition of a reactive solid by constant energy flux, Combustion Science and Technology 3, 91-98 (1971)

[7] A. Liñan and F. A. Williams, Ignition of a reactive solid exposed to a step in surface temperature, J. Appl. Math. SIAM 36, 587-603 (1979) 
[8] A. Liñan and M. Kindelan, Ignition of a reactive solid by an inert hot spot, Combustion in Reactive Systems, edited by J. Raymond Bowen, et al., Progress in Astronautics and Aeronautics, vol. 76 , American Institute of Aeronautics and Astronautics, New York, 1981, pp. 412-426

[9] N. P. Linz, Analytical and Numerical Methods for Volterra Equations, SIAM Stud. Appl. Math., vol. 7, SIAM, Philadelphia, PA, 1985

[10] R. K. Miller, Nonlinear Volterra Integral Equations, W. A. Benjamin Inc., Menlow Park, CA, 1971

[11] A. G. Merzhanov and A. E. Averson, The present state of the thermal ignition theory: An invited review, Combustion and Flame 16, 89-124 (1971)

[12] W. E. Olmsted, Ignition of a combustable half space, SIAM J. Appl. Math. 43, 1-15 (1983)

[13] F. A. Williams, Combustion Theory, 2nd Edition, Benjamin/Cummings Inc., Menlow Park, CA, 1985 